\title{
Design of Desert Plant Monitoring System Based on SI446X Wireless Radio Frequency Communication
}

\author{
Hua Guo ${ }^{1, a}$, Wenxia Zhang ${ }^{1}$, Haozhi Zhao ${ }^{2}$ \\ ${ }^{1}$ Electron Information Engineering Department of Ordos Institute of Applied Technology, \\ Kangbashi New Area, Ordos, Inner Mongolia, China. \\ ${ }^{2}$ Ordos Indemnificatory Housing Construction Management Center, \\ High Technology Industry Park, Kangbashi New Area, Ordos, Inner Mongolia, China. \\ aguohua716@163.com
}

Keywords: Desert plant, Single chip microcomputer, Wireless radio frequency.

Abstract. In this system, STH11 sensor is used to collect desert planting soil temperature and humidity what is the important environmental related data. Collected signals are transmitted to STM32F103RBT6 single chip microcomputer. Each chip is connected with a plurality of sensors to form a large scale multi point monitoring system. Using SI446X wireless radio frequency communication, lower computer of the system can make real-time detection to signals, then send the result to upper computer that records data and deals with them. This data can be used by the staff to operate. The index of desert planting is monitored in real time. This system has more measuring point, better compatibility and better expansibility, which makes the monitoring of temperature and humidity in desert plant automatic and intelligent, and improves the condition of the desert plants to die because of the lack of water, and has a positive significance to the mitigation and treatment of the desertification.

\section{Introduction}

Many of the existing environmental monitoring technology for remote environment monitoring are the use of on-site closed system, or the use of wired communication. Since the desert ecosystem is often far away from human settlements, it is very difficult to collect and obtain the data. By setting up the wired communication network, not only the equipment investment is big, the price is expensive, the communication connection is not reliable, but also can't adapt to the need of the mobile office and the remote management of the desert plant ${ }^{[1]}$. With the development of computer technology and the forestry technology, accompanied by the enhancement of forest nursing environment needs to improve, The automatic detection of the parameters, the control of the operative mechanism and various aspects of the electronic device are connected with wireless communication technology. In this way, the system can realize the automatic detection of environmental parameters, the purpose of flexible automatic control, which brings great convenience to the managers.

The main work of this system is acquisition and analysis, monitoring and control, historical data management and alarm of the data that environmental factors affecting plant growth in desert plant. The data transmission of the system is combined with the wireless radio frequency technology, and the parameters display and online parameter modification can be realized 
through the man-machine dialogue interface. By controlling the electromagnetic valve to regulate the soil moisture, the intelligent control of the desert plant monitoring system is realized.

\section{Overall Design of System}

The system adopts digital sensor STH11 to collect the temperature and humidity of the desert soil. The collected data is transmitted to the STM32F103RBT6 single chip microcomputer by the I/O interface unit. The logic operation and the processor of the single chip microcomputer compare the obtained parameters with the preset threshold of the system, and the related data are processed and stored. The processed data is transmitted to the upper computer by the SI446X wireless radio frequency module, in order to achieve the remote wireless transmission of data. The monitoring of temperature and humidity in the soil environment of desert plant growth was completed, and the upper computer returns processed control results to the single chip microcomputer through the radio frequency module. In the end, through the I/O interface and RS-232, the single chip microcomputer sends the control result to the motor, relay or electromagnetic valve, so as to realize the regulation of the soil moisture in the desert.

According to the need of monitoring system of desert plant, the overall design is shown in Figure 1. The functions of the system are as follows: The main controller, which is composed of the STM32F103RBT6 single chip microcomputer, collects the parameters such as temperature and humidity in the desert soil, and stores and processes the data; Comparison, control and feedback of the collected data, to take appropriate measures; Remote monitoring of field environmental information collection; Wireless remote transmission and remote storage of monitoring data; Distributed remote information management of multi monitoring terminal; Database management of the monitor center; man machine interactive function.

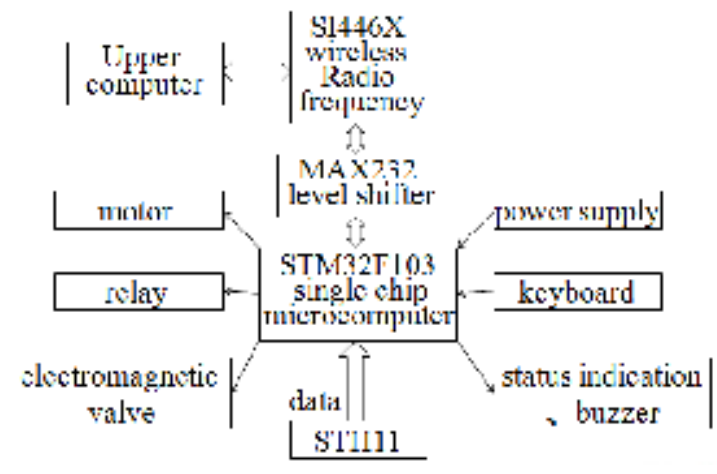

Fig.1 System block-diagram

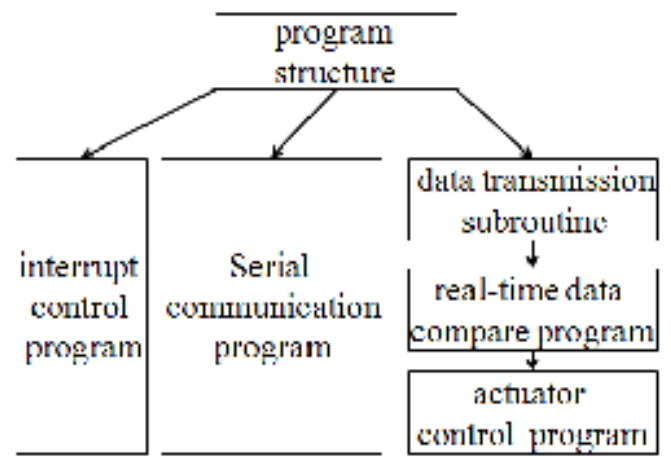

Fig.2 Software design flow chart

\section{Software Overall Design}

\subsection{Software Design of Lower Computer}

The whole program of the system was implemented as program modules that is composed with the main program and a number of independent sub program. The subroutine includes data acquisition module, data transmission module and so on ${ }^{[2]}$. The software block diagram is shown in Figure 2. System design consists of a number of independent subroutines, they are connected through the software interface, which is easy to connect and debug, but also easy to modify and transplant. The design has the larger development space; the system can 
connect multiple devices with STM32F103RBT6, so as to construct a multi-channel monitoring network. Through the development of the communication protocol of the multi-computer network monitoring, and providing a long distance communication transmission between the single chips, the data exchange of the network is realized.

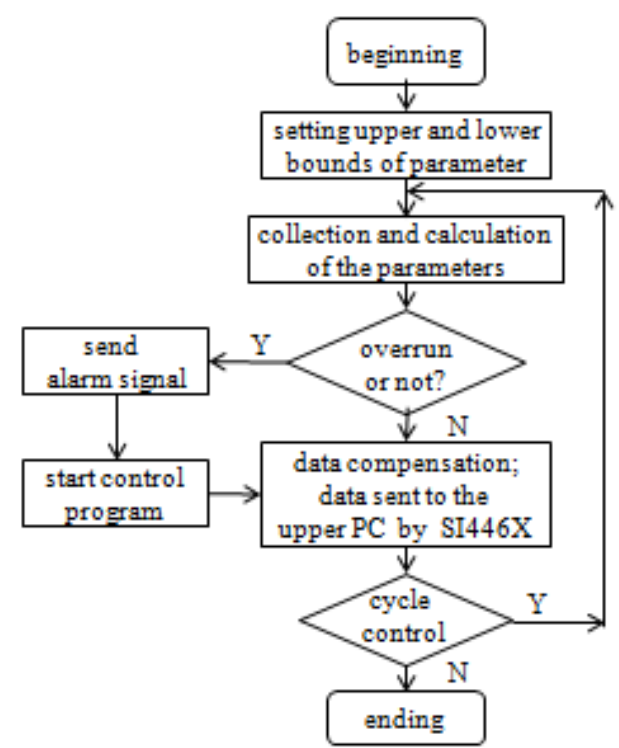

Fig.3 Lower computer software flow chart

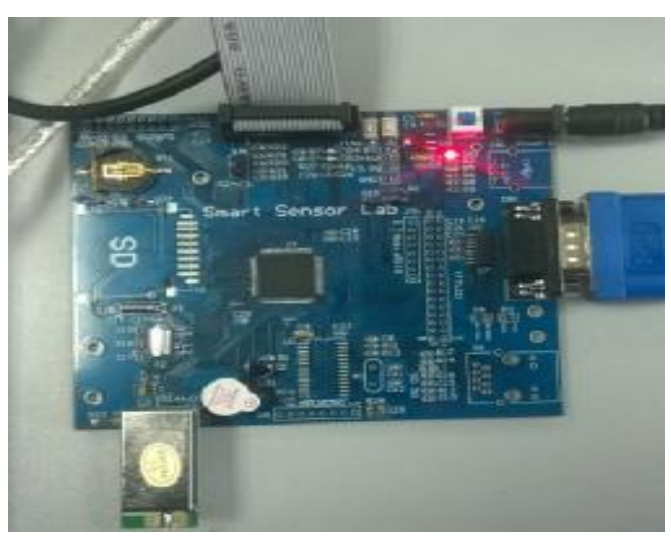

Fig.4 Host connection photo

\subsection{Software Design of Upper Computer}

The upper computer software mainly completes the processing, control, transmission of the data and communication parameters setting. Through the dedicated Iocomp ActiveX 402SP1 control, the serial port of the upper computer is connected with the single chip microcomputer or other devices conveniently and efficiently. The serial communication between PC and MCU is mainly to realize the collection, control and transmission of the lower computer data. The parameter setup of PC serial communication mainly completes the system communication parameter setting, including the baud rate setting, communication port, data bits, parity bit, the start bit and stop bit. The data processing mainly realizes the transformation, comparison, feedback and storage of the real time data, and it also prepares for the generation of the database and the data information management.

Host connection photo is shown in figure 4.

\section{Design and Implementation of Collection and Transmission}

\subsection{Design of Data Acquisition Scheme}

The design thinking is the use of distributed data acquisition system. It can be compatible with a variety of communication methods, at the same time using a number of processing systems to make the work more efficient. The mainly work of acquisition module is reading the collected digital data from sensors, then the results from the data buffer is transmitted to the stm32f103rbt6 microcontroller. In the actual data acquisition, the data's acquisition cycle should be sufficient attention. Due to the speed of the data acquisition from microcontroller and sensor is very fast, on the other hand, the change of the data in the real situation is not so fast, if 
you do not adjust the acquisition cycle, which will lead to chaos in the data collection. In order to ensure that the data can be processed timely, and the specific parameters can be controlled in real time, the method of wireless transmission is designed to control the data. After comparison, SI446X wireless radio frequency communication module is used in the system.

Each STM32F103RBT6 chip is connected with a plurality of digital output sensors to form a large scale multi point monitoring system, which improves the disadvantages that the traditional monitoring system has less measuring point, poor compatibility and poor expansibility. The field data collected by the sensors is processed by the single chip microcomputer, and then sent back to the upper computer through the wireless RF module and RS-232 to observe and store ${ }^{[3]}$. The system has the characteristics of low cost and fast cycle speed. Fig. 5 is the block diagram of the acquisition circuit.

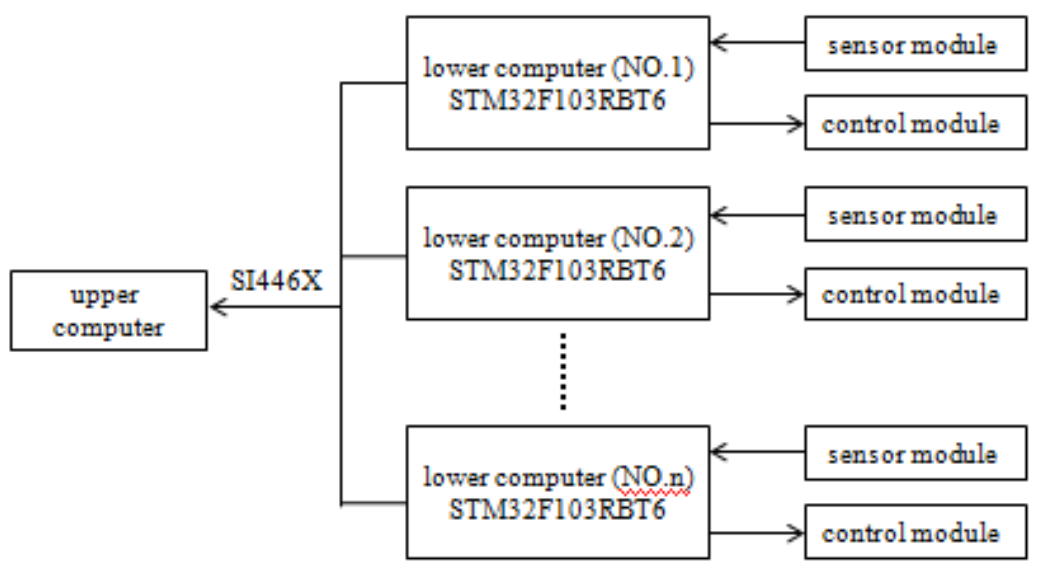

Fig.5 Data acquisition system block diagram

\subsection{Design and Implementation of Telecommunication Module}

In the system, STM32F103RBT6 single chip microcomputer is the control core, and RS-232 and radio frequency technology are combined, so as to achieve the remote control of the system equipment. As shown in Figure 6, the data of the external humidity and temperature collected by the digital sensor is transmitted directly to the single chip microcomputer. The system information is received and sent by SI446X wireless radio frequency communication module. SI446X serial communication cannot be directly connected with the RS-232, but the serial port is to follow the RS-232 interface standard, so it is through the level conversion MAX232, in order to connect with the $\mathrm{MCU}^{[4]}$. SI446X wireless radio frequency communication circuit is shown in Figure 7.

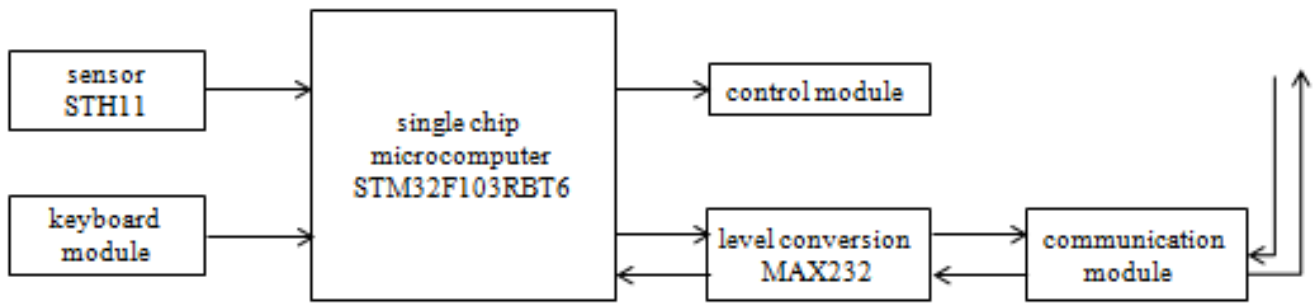

Fig.6 Communication module block diagram 

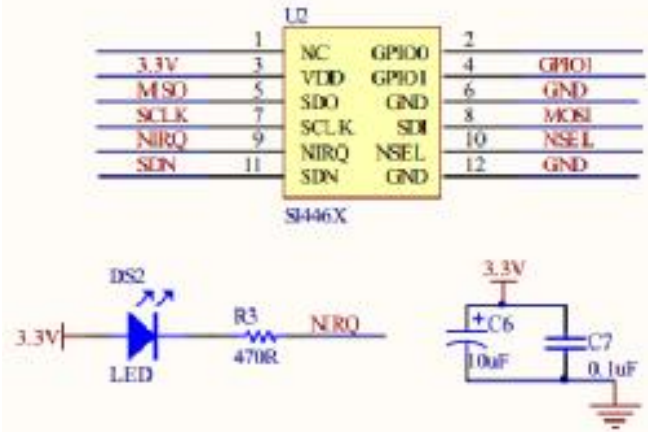

Fig.7 SI446X wireless radio frequency communication circuit

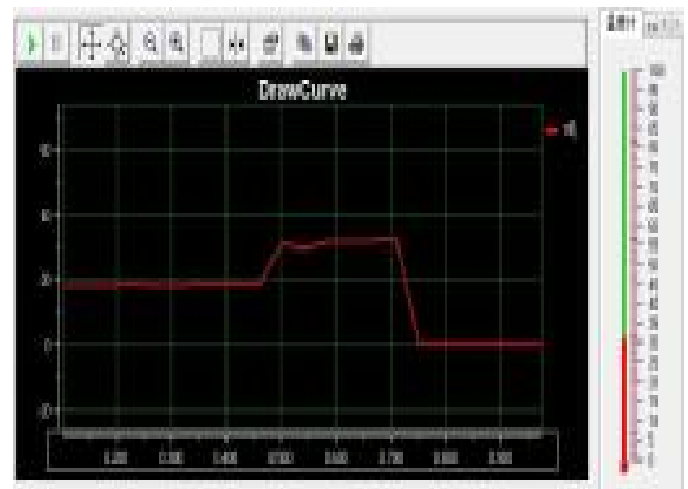

Fig.8 Temperature and humidity display window of system testing

In the first place, communication module need to write command word into single chip microcomputer, among which, the main task are to setting operating mode of the timer, serial ports initialization, the operation mode of the serial port, baud rate and interrupt bit ${ }^{[5]}$. Second, Serial transmission data frame format is set, synchronous or asynchronous communication is determined. If different data is transmitted, diacritics should be set in the start bit and the end of the data area. Also, setting the starting and end marks of control data of the upper machine is to distinguish between valid data and error code. If the data is valid, it is directly received, then in accordance with control signal order is send to control device to complete irrigation. On the other hand, if the data is incorrect, it isn't received, and the control program isn't executed, exited directly, and to wait.

Temperature and humidity display window of system testing is shown in figure 8 .

\section{References}

[1] Du Keming, Research and Implementation of Wireless Remote Monitoring System in Agricultural Environment[D], Chinese Academy of Agricultural Sciences, Beijing, 2007.

[2] Liu Ruilan, Li Ning, The Design of Intelligent Data Acquisition Terminal in Automatic Road Test System [J], Computer Technology and Development, 7 (2013)226 - 229.

[3] Zhu Kewu, He Xiaolong, Research on Greenhouse Automatic Control System [J], Agriculture Network Information, 5 (2005)52-53.

[4] Yang Jian, Research on Type Humidity Sensor and Check System Based on STM8S Microcontroller [D], Huazhong University of Science and Technology, Wuhan, 2013.

[5] Zou Difei, Research and Implementation of High-speed Wireless Sensor Data Acquisition System Based on nRF24E1 [D], University of Electronic Science and Technology of China, Chengdu, 2009. 